\title{
Fuzzy Logic Based Signal Classification with Cognitive Radios for Standard Wireless Technologies
}

\author{
Kaleem Ahmad, Uwe Meier \\ Institute Industrial IT, OWL University of Applied \\ Sciences, 32657 Lemgo, Germany \\ \{kaleem.ahmad,uwe.meier\}@hs-owl.de
}

\author{
Halina Kwasnicka \\ Wrocław University of Technology \\ 50-370 Wrocław, Poland \\ halina.kwasnicka@pwr.wroc.pl
}

\begin{abstract}
Cognitive radio (CR) is being considered as a promising technology to improve the spectral usage and coexistence behavior of radio systems. The $C R$ can work as a secondary user (SU) in coexistence with primary user (PU) systems without generating harmful interference for them. However, the performance of a SU greatly depends on its abilities to become aware of its radio environment. The more knowledge a CR can acquire from PU systems, the better it will be equipped to optimize its performance in a coexistence environment. Ideally, it would like to classify the PU systems with respect to existing 'known standards'. Research has been done in the area of signal classification with respect to modulations. We present a novel approach based on fuzzy logic (FL) to classify signals with respect to standards on the basis of known radio parameters.
\end{abstract}

\section{INTRODUCTION}

Cognitive radio (CR) offers a promising platform to realize new strategies to solve the spectrum underutilization and the coexistence problem. It operates as a secondary user (SU) by accommodating itself in available free gaps left by coexisting primary user (PU) systems in spectral, temporal and possibly some other dimensions of hyperspace [1-3]. It does so by sensing the radio environment with an objective to identify opportunities in corresponding dimensions of hyperspace. A cognitive SU aware of the identification of PU systems can better locate these opportunities in coexisting environments. Therefore the classification of incoming signals is considered important in order to improve the performance of a SU.

The process of signal classification typically consists of a feature extraction or measurement phase followed by a classification or labeling phase as shown in Fig. 1. Moreover, the classification can be done with respect to implicit signal features such as modulation, data rate, symbol rate etc. or explicit signal features such as bandwidth (BW), center frequency $\left(f_{\mathrm{c}}\right)$, signal power, time and hopping behavior etc.

Generally, the extraction of implicit signal features needs complex and expensive signal processing. For instance, a popular method for this purpose is the computation of the spectral correlation function (SCF) which requires the FFT computation of order $N$ followed by cross-correlation of order $\mathrm{N}^{2}$ [4]. Such challenging computational effort is not feasible in existing radio systems. On the other hand explicit signal features can be extracted without going into internal details of the signal e.g. by using power spectral density (PSD) or frequency-time representations of signals. It merely needs FFT processing of order $N$.

Signal classification is already a popular research topic and computational classifiers such as support vector machine
(SVM), or statistical classifiers such as histograms, Bayesian networks and hidden Markov models (HMM), or connectionist classifiers such as neural networks $(\mathrm{NN})$ are mostly used for this purpose. In addition to these methods, case and rule based reasoning is also occasionally used. A survey of radio signal classification methods is presented in table I.

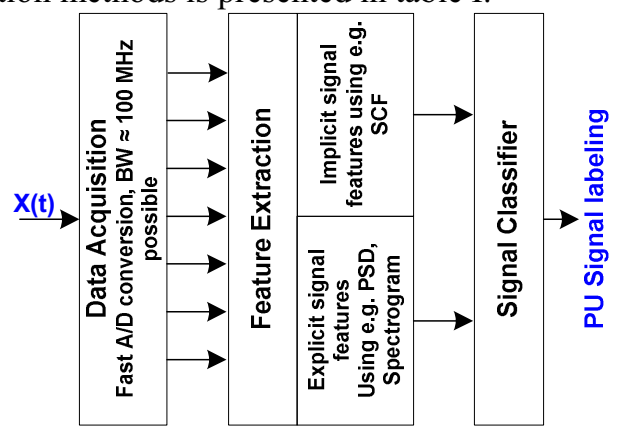

Figure 1: Signal classification process

Despite the strengths and weaknesses of individual classification methods, following major shortcomings are common in most of these strategies:

1. Most frequent used classifiers are based on NN, SVM, and HMM which require high number of data samples for training purpose. It puts a performance limit in terms of cost and time. Furthermore, generalization remains a critical issue in such strategies i.e. how well will the classifier make classification of patterns that are not in the training set and eventually such classifiers can suffer from either underfitting or over-fitting.

2. Class labeling is limited to modulation. However, mere modulation classification doesn't provide sufficient information to find coexistence opportunities since several radio systems may employ a single modulation strategy but very different behavior from the coexistence perspective. For example, both Bluetooth (BT) and Atmel ATR2406 operate in the $2.4 \mathrm{GHz}$ ISM band and implement GFSK modulation. However, the ATR2406 system operates in a narrow frequency range and is easy to detect and accommodate in a coexisting environment [1]. Whereas the BT system hops over 79 channels and pose real challenge to SU systems in terms of detection and finding opportunities in its coexistence. Therefore, it is necessary to classify the PU signals with respect to known standards (e.g. IEEE 802.11 WLAN, IEEE 802.15.1 BT, etc) so that the documented knowledge of PU radio systems can be utilized. 
Table I: A survey of radio signal classification methods

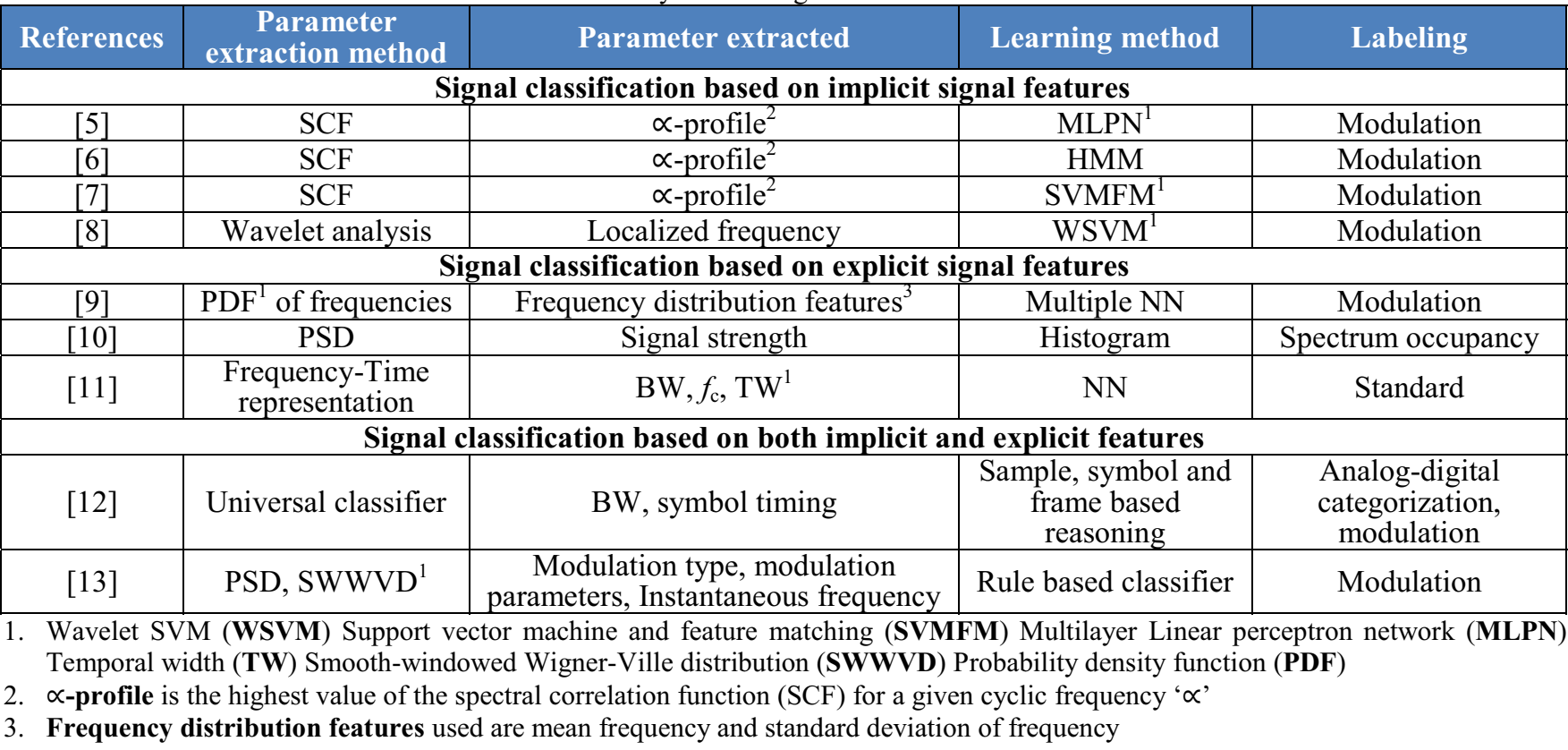

We present a fuzzy logic (FL) based signal classification strategy which labels the PU signals with respect to known standards. Since the presence of noise, multipath, Doppler spread, and coexisting effects makes it difficult to categorically identify the PU signals therefore FL can be an excellent choice to implement a radio signal classifier since it provides a simple way to arrive at a human like conclusion based upon vague, noisy or missing input information.

The use of FL has already gained some attention in CR research. For instance, a transmit power control system using FL to provide cognitive radios the ability to coexist with PUs is presented in [14] and the use of FL for the representation of cross-layer information and the implementation of optimization strategies in CR systems is studied in [15]. The simplicity of the system model in such studies is a spin-off source of motivation to choose FL for signal classification.

Furthermore, our strategy doesn't need lot of data, time and complex algorithms for training unlike existing classifiers. It is efficient since it relies on explicit signal features though it can easily be extended to include implicit signal classifier as soon as sufficient computation resources will be available in radio systems. For the proof of concept we choose $\mathrm{BW}, f_{\mathrm{c}}$ and hopping/time behavior as distinct features to classify PU signals. Moreover, we demonstrate the performance of proposed idea by classifying some well known standard technologies in $2.4 \mathrm{GHz}$ ISM band.

\section{FUZZY LOGIC BASICS}

FL deals with uncertainties and ambiguities in a way that mimics human reasoning. FL based systems are conceptually easy to understand and the mathematical concepts behind their reasoning are very simple. Furthermore, the truth of each statement is a matter of degree. The rule-based decision is the heart of a FL system and contains the set of if-then rules. Such rule sets are flexible and new rules can easily be added to extend the functionality of the system. For example, the following simple rule can be used to classify some well-known non-hopping radio systems having distinct $\mathrm{BW}$ and/or $f_{\mathrm{c}}$ in the $83 \mathrm{MHz}$ wide $2.4 \mathrm{GHz}$ ISM band.

if BW matched BW PUi $_{\text {AND }} f_{\mathrm{c}}$ matched to one of channel $_{j^{\text {th }}}$ of $P U i$ then $P U$ i operating in $j^{\text {th }}$ channel

The following new rule, to analyze the hopping behavior of signals, can be added without changing the exiting functionality of the classifier.

if BW matched $1 \mathrm{MHz}$ AND $f_{\mathrm{c}}$ is hopping

then $\mathrm{BT}$ based $P U$ is operating

Please note that all bold italic words are fuzzy or linguistic variables defined over some base variable. The set of values that it can take is called the term set. For instance a term set $T$ for fuzzy variable 'matched' can be defined on the base variable $\mathrm{BW}_{P U i}-\mathrm{BW}$ as follows:

$T=\left\{\begin{array}{c}\text { matched, strongly matched, almost } \\ \text { matched, weakly matched, not matched }\end{array}\right\}$

In order to understand the functionality of the proposed model it is necessary to understand how radio world concepts are mapped to FL concepts as described in the following.

Fuzzy set: A fuzzy set is a collection of ordered pairs as follow:

$A=\{(x, \mu(x))\}$

Where item $x$ belongs to the universe of discourse and $\mu(x)$ is the degree or grade of membership. Since frequency is the central resource in radio environment and all electromagnetic activities revolves around it, we can replace $x$ with frequency $f$ to define our fuzzy radio set (FRS), as follows:

$F=\{(f, \mu(f))\}$ 
Universe of discourse: The input space, where elements of a fuzzy set are taken from, is called universe of discourse or simply universe and is often represented by $u$ or $U$. The entire radio frequency is the universe in our fuzzy radio model (FRM).

Membership function (MF): A membership function (MF) is a curve that defines how each point in the input space is mapped to a membership value (or degree of membership) between 0 and 1 . It is conventionally denoted as $\mu(x)$ or equivalently as $\mu(f)$ in FRM. The set of elements that have a non-zero membership is called the support of the fuzzy set

Standard Fuzzy Operators: Boolean operators AND, OR, and NOT are interpreted as intersection or min $(\cap)$, union or $\max (U)$, and fuzzy complement $(1-A)$ respectively.

Similarity measure $(\boldsymbol{S M})$ : Similarity measure is used in fuzzy mathematics to measure the grade of similarity between two fuzzy sets. Several definitions of similarity measure have been presented. We adapt the similarity measure $S M$ between fuzzy sets $A$ and $B$ defined in [16]. It is based on the minimum relative sigma count of $A$ in $B$ (and $B$ in $A$ ) as follows:

$S M(A, B)=\frac{|A \cap B|}{\max (|A|,|B|)}$

Or equivalently:

$S M(A, B)=\frac{\sum_{i=1}^{n} \min \left(\mu_{A}\left(f_{i}\right), \mu_{B}\left(f_{i}\right)\right)}{\max \left(\sum_{i=1}^{n} \mu_{A}\left(f_{i}\right), \sum_{i=1}^{n} \mu_{B}\left(f_{i}\right)\right)}$

\section{Fuzzy Signal Classifier}

We choose BW and $f_{\mathrm{c}}$, as distinct features to classify PU signals. Our strategy is not limited to the classification of some specific PU systems however, we choose IEEE 802.11 WLAN, IEEE802.15.1 BT, and FSK based Atmel's ATR2400 as PUs to explain and demonstrate our idea. The later system will be simply referred as FSK in the remaining text. Important features of these PUs are given in table II while the algorithm used to classify the PU signals is shown in table III.

Table II: Important features of PU systems

\begin{tabular}{|c|c|c|c|c|}
\hline & BW & $\boldsymbol{f}_{\mathbf{c}}$ & Hopping & Freq. Band \\
\cline { 1 - 4 } WLAN & $22 \mathrm{MHz}$ & 13 channels & no & \multirow{2}{*}{$2.4 \mathrm{GHz}$} \\
\cline { 1 - 4 } BT & $1 \mathrm{MHz}$ & 79 channels & yes & ISM band \\
\cline { 1 - 4 } FSK & $0.864 \mathrm{MHz}$ & 95 channels & no & \\
\hline
\end{tabular}

The idea is to fuzzify the incoming PSD and filter it over certain $f_{\mathrm{c}}$ and $\mathrm{BW}$. These filtered segments $\left(F P S_{i, j}\right)$ of the fuzzified PSD $\left(F P S_{\mathrm{U}}\right)$ can be compared with the predesigned ideal power spectrum $\left(I S_{i, j}\right)$ of known PU systems at that particular $f_{\mathrm{c}}$. The presence of a specific PU signal can then be labeled on the basis of comparison results $\left(S M_{i, j}\right)$. Important components of this algorithm are explained in the following sections.

Fuzzy Power Spectrum (FPS): A real-time spectrum analyzer (Tektronix RSA 6114A) is used to acquire the entire $2.4 \mathrm{GHz}$ ISM band at $150 \mathrm{MS} / \mathrm{s}$ sampling rate. It generates a $83 \mathrm{MHz}$ wide PSD which is further sampled at $166 \mathrm{kS} / \mathrm{s}$ sampling rate to obtain 501 point $U_{f}$ for post processing in Matlab. Hence universe of discourse is as follows:
$U_{f}=\{2400 \mathrm{MHz} \leq f \geq 2483 \mathrm{MHz}\}$

The received power $P_{f}$ at each frequency point of interest can be used to fuzzify the PSD to $F P S_{\mathrm{U}}$ using the following membership function:

$\mu(f)=\left|\frac{P_{\min }-P_{f}}{w}\right|$

Where $P_{\min }$ is the minimum expected power and $w$ is the total range of the expected received power distribution $\left(w=P_{\min }-P_{\max }\right)$. An example $F P S_{\mathrm{U}}$ is shown in the upper plot of Fig. 2. The universe of discourse is represented as $F P S_{U}$ and a filtered segment of universe using $i^{\text {th }}$ PU's $j^{\text {th }} F S$ is represented by $F P S_{i, j}$ in algorithm shown in table III.

Table III: Algorithm used for standard classification

1 Design fuzzy stencils $\left(F S_{n, m}\right)$ and ideal spectrums $\left(I S_{n, m}\right)$, where $n$ represents the number of the corresponding PU which has $m$ predefined channels. The value of $m$ is different for each PU system.

2 Fuzzify input PSD: $F P S_{\mathrm{U}}$

3 Initialize: $i=0, j=0, k=0, S M_{n, m}=0$ While $i \leq n$

While $j \leq m$

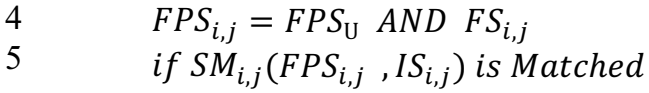

Then PU $i$ is Operating in channel $\mathrm{j}$
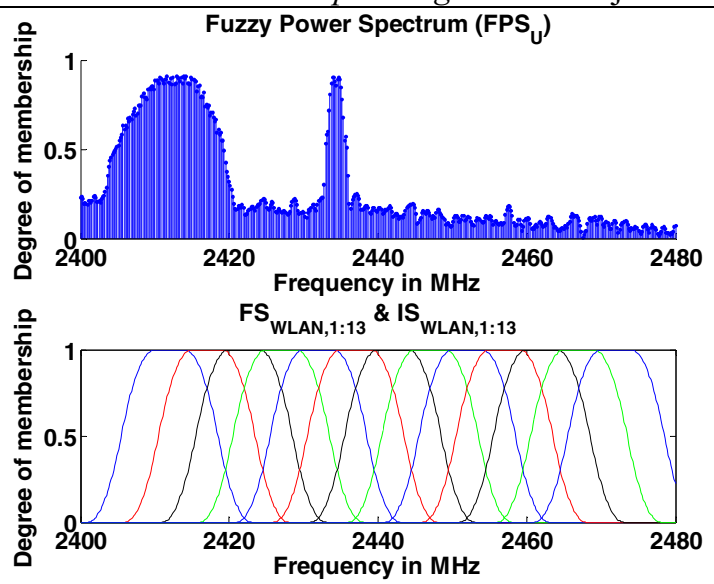

Figure 2: The $F P S_{\mathrm{U}}$ is shown in upper plot. A WLAN and a FSK system are operating. Lower plot shows $F S$ and $I S$ sets

Fuzzy Stencil (FS): A set of curves, named as $F S$, is designed using the center frequencies of corresponding PU channels and the bandwidth of the widest of all PUs of interest. For instance $\mathrm{BW}_{\mathrm{WLAN}}=22 \mathrm{MHz}$ will be used as $\mathrm{BW}$ of $F S$ for all PUs in our experiments. The set $F S$ is used to cut or filter a segment of $F P S_{\mathrm{U}}$ (step 4 in table III). This process extracts the features $\mathrm{BW}$ and $f_{\mathrm{c}}$ so that the classifier can check for the presence of a certain PU. The FS for the FSK system is given in the following as an example. 
$F S_{\mathrm{FSK}}=\left\{\begin{array}{c}\pi-\operatorname{Curve}\left(\mathrm{BW}, f_{c}\right): \mathrm{BW}=22 \mathrm{MHz} \text { and } \\ f_{c}=[\text { of } 95 \mathrm{FSK} \text { channels }]\end{array}\right\}$

The reason to choose the BW of $F S$ for all PUs equal to the BW of widest PU system is the fact that choosing a narrower stencil to detect a narrow band signal, where a wider band PU is actually operating, will always incorrectly results in a perfect match for narrowband signals. For instance, if we filter the $F P S_{\mathrm{U}}$ shown in Fig. 2 using $F S_{\mathrm{FSK}, 14}=0.864 \mathrm{MHz}$ at $2412.3 \mathrm{MHz}$ and check for the presence of a FSK signal then the classifier will incorrectly suggest the presence of a FSK signal, although it is a wider WLAN signal. However, if we keep the $\mathrm{BW}$ of $F S_{\mathrm{FSK}}$ equal to the $\mathrm{BW}_{\mathrm{WLAN}}$ then the denominator factor of $S M$ will be too big, keeping the value of $S M$ small, when the narrow band FSK will be compared at frequencies where a WLAN system is actually operating (see Fig. $3 \& 4$ for further explanation.)

Ideal Spectrum (IS): It is a set of best possible approximation of the power spectrum of the PU of interest over its predefined channels. Similarity measure of filtered $F P S_{\mathrm{U}}$ at a certain $f_{\mathrm{c}}$ is computed (step 5 in table III) with corresponding $I S$ in order to detect the presence of that PU. The IS for FSK channels is defined as follows.

$I S_{F S K}=\left\{\begin{array}{c}\pi-\operatorname{Curve}\left(\mathrm{BW}, f_{c}\right): \mathrm{BW}=0.864 \mathrm{MHz} \text { and } \\ f_{c}=[\text { of } 95 \mathrm{FSK} \text { channels }]\end{array}\right\}$

The sets $F S$, and $I S$ are the same only for the widest PU as shown for WLAN systems in the lower plot of Fig. 2.

\section{A WORKING EXAMPLE}

The classifier obtains the $F P S_{\mathrm{U}}$ shown in Fig. 2 as input and the output of each step for both WLAN and FSK signals is shown in Fig. 3 and Fig. 4 respectively. Only two elements of $F S, I S$ and filtered $F P S_{\mathrm{U}}$ i.e. $F P S_{i, j}$ are plotted in parts a) \& b) of these figures to improve the readability. The membership function to grade $S M$ is shown in Fig. 5. It can be seen from the $S M$ plots (Fig. 3c, Fig. 4c) that a WLAN signal is correctly matched at Channel WLAN,1 $_{1}$ and a FSK signal at Channel $_{\mathrm{FSK}, 39 \& 40}$.

Fuzzy spectrogram (FSG) for classification of similar signals: The presence of more than one $\mathrm{PU}$ with highly identical channel $\mathrm{BW}$ and $f_{\mathrm{c}}$ requires the analysis of further distinct signal features. For instance, both BT and FSK systems are highly identical with respect to these two parameters. The $S M$ measured over a single $F P S_{\mathrm{U}}$ for any of these systems will include incorrectly identified strong other PU signals. However, the $S M$ measured over several $F P S_{\mathrm{U}} \mathrm{S}$ can help to generate a separate FSG for each PU which in turn can be used to study the hopping and time behavior of signals. An example FSG for the BT system measured over four $F P S_{\mathrm{U}} \mathrm{S}$ is shown in Fig. 6. It can be seen that the FSK signals are also detected as BT signals. However, a statistical dispersion analysis of FSG will help to distinguish between 'the static behavior of FSK and hoping behavior of BT' signals.
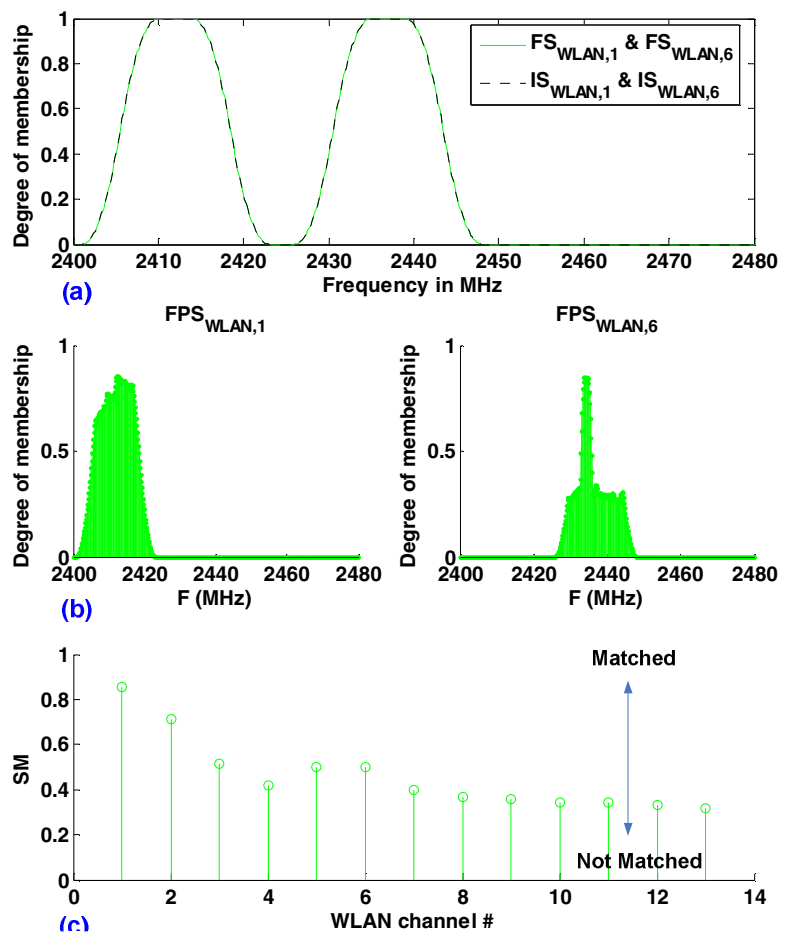

Figure 3: WLAN signal detection. a) 2 of 13 fuzzy stencils and ideal spectrums of WLAN system. b) the FPS filtered using $F S_{\mathrm{WLAN}, 1} \& F S_{\mathrm{WLAN}, 6}$. c) $S M$ for all 13 WLAN channels
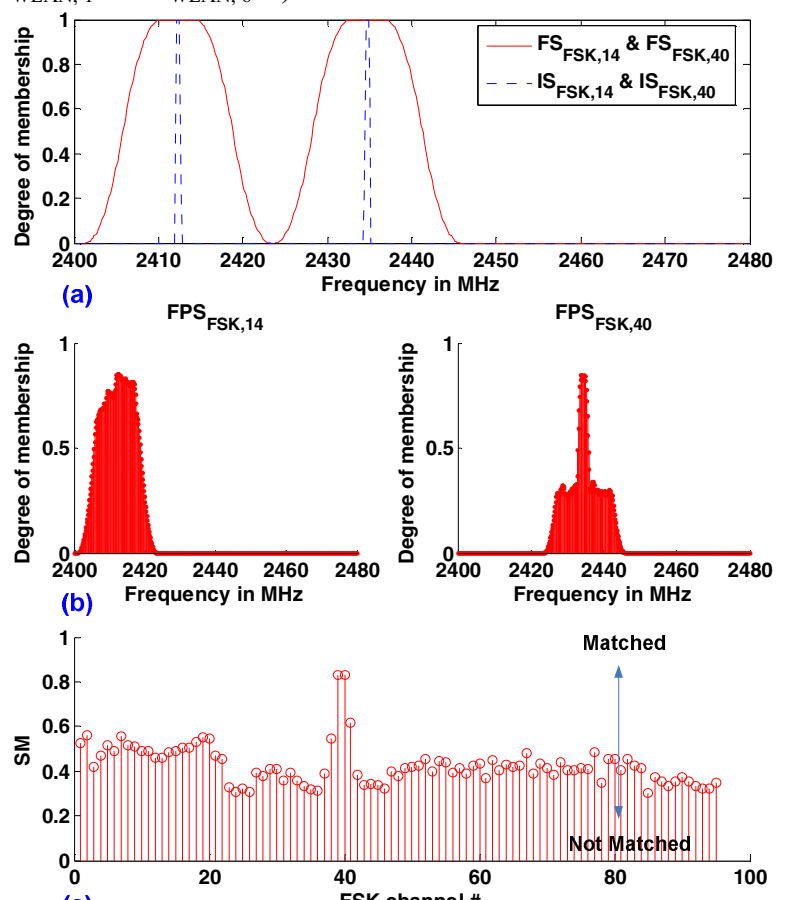

(c)

Figure 4: FSK signal detection. a) 2 of 95 fuzzy stencils and ideal spectrums of FSK system. b) the FPS filtered using $F S_{\mathrm{FSK}}$, ${ }_{14} \& F S_{\mathrm{FSK}, 40}$. c) $S M$ for all $95 \mathrm{FSK}$ channels 


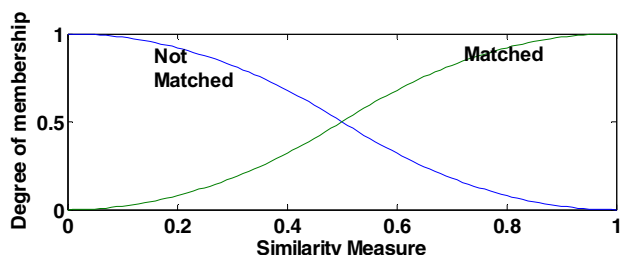

Figure 5: The MS function to grade the $S M$

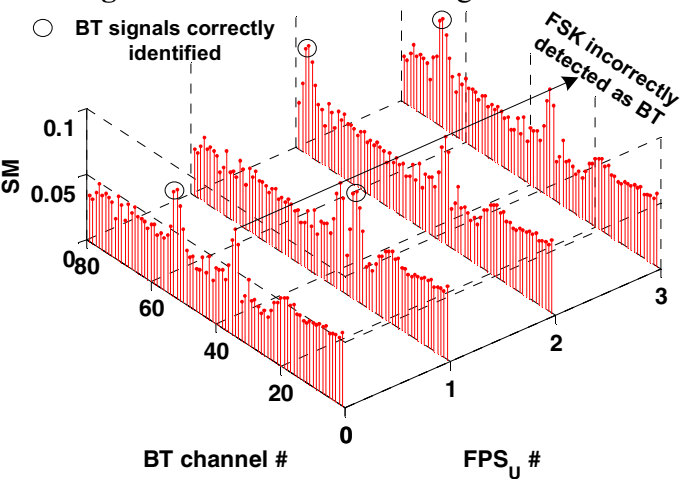

Figure 6: The Fuzzy Spectrogram for BT system

Detection of weak PU signals: In order to improve the prominence of weaker signals while observing them with a spectrum analyzer one needs to adjust the reference level. At the same pattern, the FL based classifier can increase the chances of identification of weaker PU signals by improving the prominence of such weaker peaks during the fuzzification process by merely adjusting the range parameter ' $w$ ' of Eq. 3 .

For instance, the following parameters are used to achieve $F P S_{\mathrm{U}}$ as shown in Fig. 2 from a PSD which contain WLAN and FSK signals with peak amplitudes in the order of $-55 \mathrm{dBm}$.

$P_{\text {min }}=-90 \mathrm{dBm}, P_{\max }=-50 \mathrm{dBm} \rightarrow w=40$

It results as 'strongly matched' WLAN and FSK signals. However, when the same signals are weaker by a factor of $7 \mathrm{~dB}$, i.e. the peak amplitude is in the order of almost $-62 \mathrm{dBm}$ for both signals, the following parameters can be selected to stretch the weaker PU signal peaks in fuzzy interval $[0,1]$ and to level $S M$ at almost the same value or even better than that of its $7 \mathrm{~dB}$ stronger counterpart as shown in Fig. 7.

$P_{\min }=-90 \mathrm{dBm}, P_{\max }=-60 \mathrm{dBm} \rightarrow w=30$

or

$P_{\text {min }}=-82 \mathrm{dBm}, P_{\max }=-60 \mathrm{dBm} \rightarrow w=22$

\section{CONCLUSION}

The classification of PU signals with respect to standards can improve the performance of SU systems since the documented knowledge of PU radio systems can be usefully utilized. We propose a FL based signal classification strategy which extracts $\mathrm{BW}$ and $f_{\mathrm{c}}$ of PU signals from measured PSD information to label the signals with respect to standards. Fuzzy spectrograms can be generated by combining $S M$ vectors measured over multiple $F P S_{\mathrm{U}}$ s to study the hopping and/or time behavior to distinguish between signals, which are either highly identical in terms of $\mathrm{BW}$ and $f_{\mathrm{c}}$ or overlapping in spectral axis. Furthermore, the fuzzification process can improve the prominence of weaker PU signals by merely adjusting the reference amplitude.
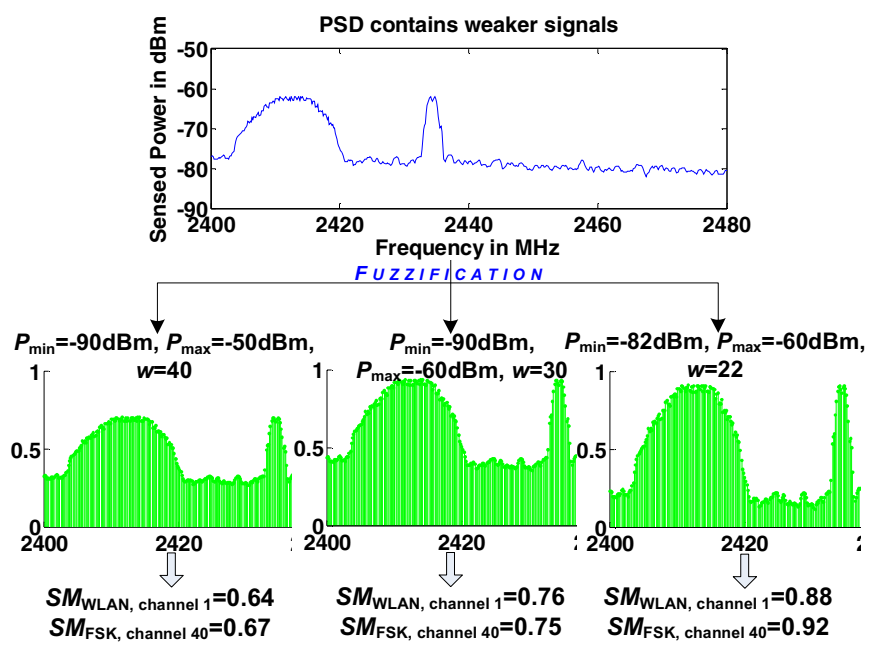

Figure 7: Weaker peaks are made prominent during fuzzification process, which results in better signal detection.

\section{REFERENCES}

[1] Ahmad, K; et al., "A cognitive radio approach to realize coexistence optimized wireless automation systems" ETFA 09.

[2] http://www.ieee802.org/22/

[3] Geirhofer, S.; et al., "Cognitive radios for dynamic spectrum access - dynamic spectrum access in the time domain: modeling and exploiting white space”, IEEE Comm Magazine, May 2007.

[4] Tkachenko, A. et al.; "Cognitive Radio Experiments using Reconfigurable BEE2,” ACSSC '06, Oct. 29 2006-Nov. 12006

[5] Fehske, A. Gaeddert, J. Reed, J.H.; "A new approach to signal classification using spectral correlation and neural networks", DySPAN, 11 Nov. 2005

[6] Kim, K.; et al.;"Cyclostationary Approaches to Signal Detection and Classification in Cognitive Radio", DySPAN 2007

[7] Hang Liu; et al.; "A New Approach to Improve Signal Classification in Low SNR Environment in Spectrum Sensing," CrownCom 15-17 May 2008.

[8] D. Wu, et al. "A New Scheme of Automatic Modulation Classification Using Wavelet and WSVM," MTAS 2005.

[9] Cattoni, Andrea F.; et al.; "Neural networks mode Classification based on Frequency Distribution Features," CrownCom 2007

[10] J. Mitola, "Cognitive Radio Architecture," 2006 by John Wiley \& Sons, Inc.

[11] Mody, A.N.; et al.; "Recent advances in cognitive communications," IEEE Communications Magazine, Volume 45, Issue 10, October 2007 Page(s):54 - 61

[12] Qinqin Chen; et al.; "Universal Classifier Synchronizer Demodulator," IPCCC 7-9 dec. 2008.

[13] Jo Lynn Tan; et al.; "Signal analysis and classification of digital Communication Signals using Adaptive Smooth-Windowed Wigner-Ville Distribution," NCTT-MCP 26-28 Aug. 2008.

[14] Le, H.-S.T.; Qilian Liang; "An Efficient Power Control Scheme for Cognitive Radios," WCNC 2007.

[15] Nicola Baldo, et al.; "Fuzzy Logic for Cross-layer Optimization in Cognitive Radio Networks," IEEE Communications Magazine, April 2008.

[16] Hassan Rezaei, et al,; "New Similarity Measure Between Two Fuzzy Sets," Journal of Advanced Computational Intelligence and Intelligent Informatics. Vol.10 Nov. 2006 